\title{
Melanoma Diagnostic System using Non-Shannon Havrda Measure and Harris Corner Detector
}

\author{
Neena Agrawal \\ M. Tech \\ 38, A, Sanjay Nagar, Niwaru Road, Jhotwara \\ Jaipur-302012, Rajasthan (India)
}

\author{
Vineet Khanna \\ Asst. Professor \\ Rajasthan College of Engineering for Women \\ Jaipur
}

\begin{abstract}
Malignant Melanoma is the deadliest type of skin cancer, is one of the most rapidly increasing cancers in the world. Malignant Melanoma is very difficult to treat and it must be diagnosed and excised during its earliest stages. In this work, we use a data set of 184 clinical dermatoscopic images of skin lesions, in which 144 images are of malignant lesion and 40 images are of benign lesion. we classify skin lesions as malignants from color photographic slides of the lesions. So we use color images of skin lesions, image processing techniques and artificial neural network to distinguish from benign pigmented lesions. In image processing we use Image level median filtering and entropy based segmentation technique. At the first step, we Consider clinical images of skin cancer patients using high speed cameras. After that we uses Medial filtering and histogram preprocessing to avoid uneven illuminance problem. Then Harris Corner detection method is implemented to characterize edges of the image for further analysis and implementation. Second, Otsu and Entropy based image segmentation algorithm is performed which improves the quality of the image. Its used for lesion extraction. In this work we use Machine learning based approach to optimize the classification error. In this we have instigated the performance of entropy based method against standard Otsu method and proved that the information theory based non-shannon entropy function of Havrda give far optimum performance against histogram based approach.
\end{abstract}

\section{Keywords}

Component; formatting; style; styling; insert (key words)

\section{INTRODUCTION}

Cancer has a time period for ailments where the body's cells begin to divide without stopping and spread into surrounding tissues. There are over two hundred different kinds of cancer, and every is classified by the type of cell that is initially affected. Six characteristics of malignancies had been proposed:, proliferative signalling, evading retaining development suppression, withstand cell dying, replicative immortality enabling, angiogenesis inducing and activating invasion and metastasis can form an instantaneous mass-time melanoma cells from typical cells progress that includes many steps. Cancer cells can unfold to other components of the physique through the blood and lymph methods. When cancer develops, however, this orderly methods breaks down. As cells become more and more abnormal, old or broken cells survive oce they ought to die, and new cells form when they are not required. These extra cells can divide without stopping and may form growths called tumors.

Now not all tumours are cancerous; Tumours may also be benign and malignant. The reasons of cancer are complex, various, and understood in part most effective. There are quite a lot of matters which are known to increase the chance of melanoma, including special infections, exposure to radiation, lack of physical endeavor, obesity, tobacco use, dietary causes, and environmental pollutants.

In traditional cell DNA. When it broken, the cell either repairs the injury or dies. But in cancer cells, the damaged DNA neither repairs the injury nor dies. Alternatively, it offers upward thrust to more of the abnormal cells containing abnormal DNA. These new cells all have the equal defective DNA of the usual cancer cell.

Benign tumours: - Benign tumours are not cancerous. In this, it can often be removed and do not come back. It does not spread to other parts of the body.

Malignant tumours:- Malignant tumours are cancerous. they can spread into, or invade, nearby tissues. The spreading up of cancer from one body part to another is called metastasis[2].

Melanoma is not just one disease however has many ailments. There are special varieties of melanoma and which can also be grouped into broader categories.

\section{STUDY OF PRIOR ARTS}

In various image domains many approaches has been carried out by scholars targeted for a melanoma feature analysis and classification. In [1], awarded asymmetry, border irregularity, color variation, and diameter (ABCD) function extraction of dermatoscopic image for melanoma epidermis cancer diagnosis. Asymmetry characteristic includes the knowledge about asymmetry and lengthening index of the lesion. Expertise of compactness index, fractal dimension, area abruptness, and pigmentation transition from the lesion are included in Border irregularity characteristic. In [2], validated in his study that MATLAB is a strong software for the early prediction and prognosis of malignant melanoma by utilising numerical image processing procedures. In [4], We evaluate different segmentation methods which utilized on the microscopic crystal photograph. Segmentation by means of watershed distance turn out to be in each the non-overlapping and the problem arises in overlapping objects in the photograph and A drawback arises when we composed the vicinity by using multiple elements and separation discipline of two overlapping objects must be considered due to the fact that most of them have a line for separation, nonetheless resolution of this line is valuable for the whole process. In [5], proposed a procedure to classify skin lesions as malignant or benign from color photographic slides of the lesions. For distinguishing melanoma from benign pigmented lesions, $\mathrm{He}$ makes use of color portraits of epidermis lesions, snapshot processing techniques and synthetic neural community classifier. In proposed the ABCD score calculation algorithm and implemented on an Android-headquartered cellular platform. He firstly identifies and localizes dermis blemishes in a larger skin picture making use of the change-of-Gaussians 
and SVM detector. In [1], evaluated that the images showed beneath noisy stipulations Canny, LoG( Laplacian of Gaussian), Robert, Prewitt, Sobel showcase better performance, respectively and showed that Canny's detection algorithm is computationally more costly in comparison with LoG (Laplacian of Gaussian), Sobel, Prewitt and Robert's operator. In [2], places emphasis on the learning method, trying out the awareness efficiency of three one-of-a-kind classifiers: SVM, synthetic neural community and k-nearest neighbour. Huge experiments have been run on a database of more than 5000 dermoscopy portraits. The obtained outcome exhibit that the SVM method outperforms the opposite methods

\section{MELANOMA DIAGNOSTIC MODEL}

In this work we targeted to discover malignant lesion from the given pattern images. The predominant purpose of the proposed skin melanoma diagnostic system is extraction of features of the photograph, making use of pre-processing and segmentation techniques so that you could enhance the highquality of the picture after which evaluate the points of the photo which are Asymmetry (A), Boundary (B), color editions (C) and Diameter (D) for the detection of malignant and nonmalignant melanoma. The procedures required to include is depicted in fig. 1 whose details are as follows:

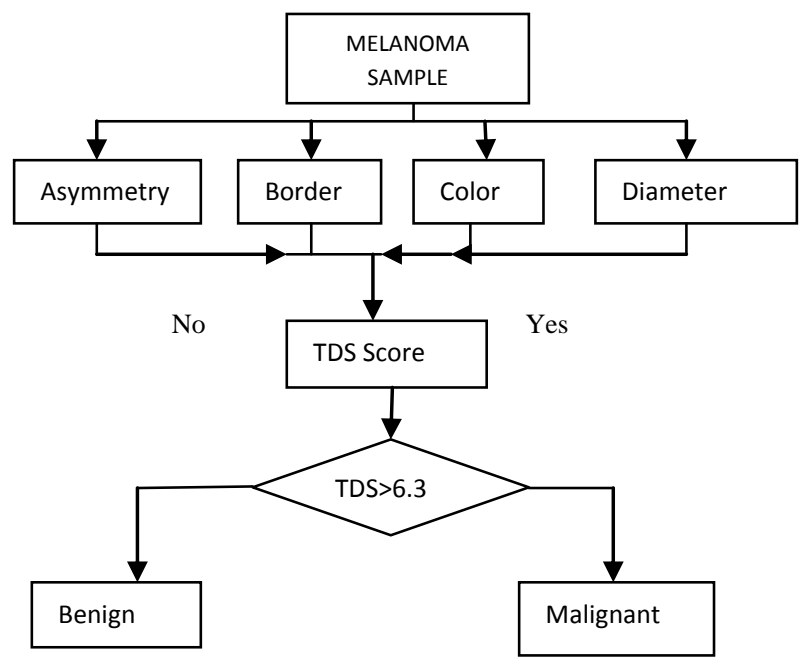

Fig.1: Flow Diagram of Melanoma Detection and Classification

\subsection{Image Pre-Processing}

First step is Hair and Noise elimination from the photograph. In this Median filtering is used to minimizing presence of small buildings like hairs. In image processing, it's quite often fascinating to be able to participate in some form of noise reduction on an image. The median filter is a nonlinear digital procedure, almost always used to take away noise.

\subsection{Melanoma Segmentation}

In image segmentation approach an image segments into its constituent regions or object. This procedure is based on a threshold price to transform in to a gray-scale photo right into a binary picture.

In this subsection, we discuss the distinct entropy measures [3], [8]-[9], which can be used in this thesis for comparative gain knowledge of in photograph segmentation issues. In this we the methodology of snapshot segmentation using the gray level co-occurrence matrix $\left(C_{m_{1}, m_{2}}\right)$ and Shannon entropy measure [5]. In this work we extend this technique making use of the co-occurrence matrix with non- Shannon entropy measures (reminiscent of Renyi, Havrda-Charvat, Kapur and Vajda entropy) on colour graphics. The non Shannon entropy functions are inherited from[6]. The segmentation results of the algorithm are reproduced right here for the sake of convenience in Table.1:

TABLE I. LESION Extraction USING Shannon, NonSHANNON \& OTSU APPROACH

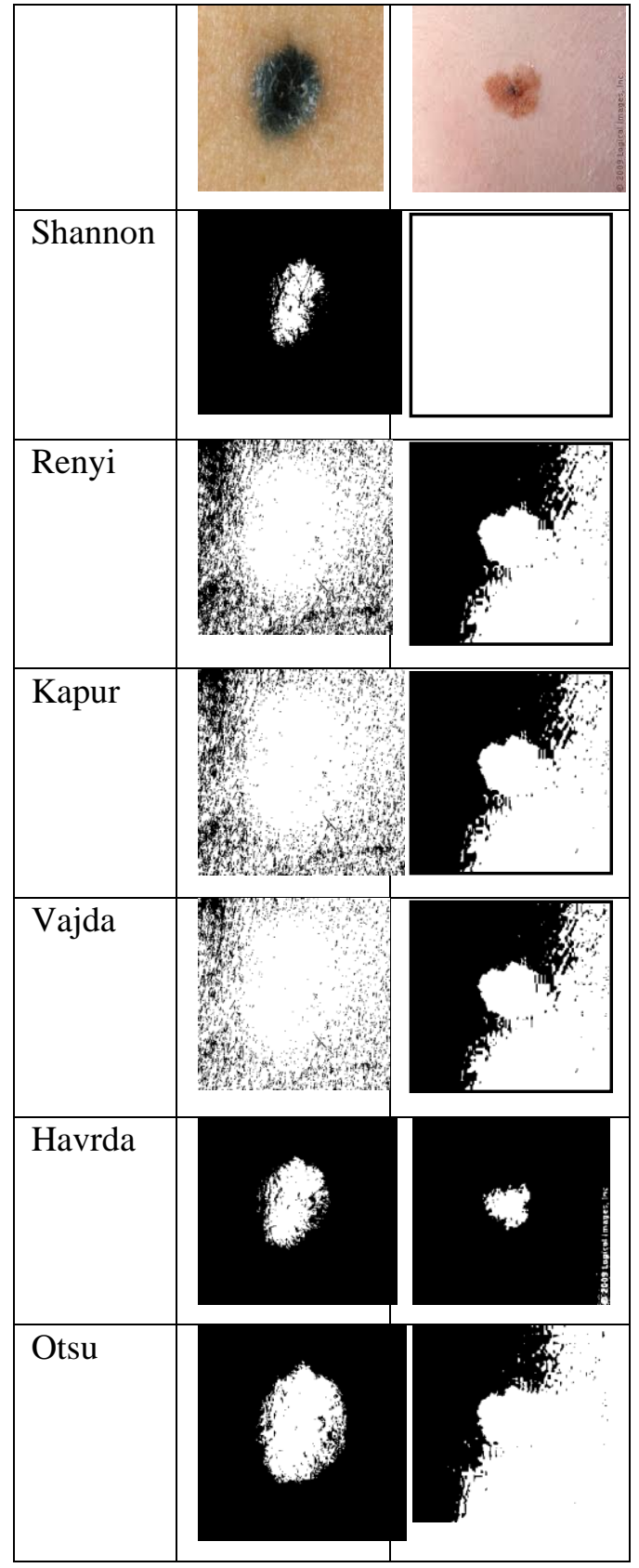

The Havrda enropy based approach gives more accurate results in terms of retaining the lesion shape, size and border irregularities.

\subsection{Malignant Features of Melanoma}

In this section compares the features, i.e., visual cues which are used for melanoma detection. After the evaluation of the entire features, we are able to characterize the lesion as malignant or non-malignant. The features used for the diagnostic method for 
the detection of melanoma are Asymmetry (A), Border (B), Color (C), Diameter (D) and Evolving (E).

\subsubsection{Asymmetry}

To find the degree of asymmetry we calculate the circularity of the shape. For this we calculate the area (A) and perimeter (P). The lesion is divided into four parts by the major and minor axis which crosses in the centre of the lesion. We calculate the area difference across each axis with the formula:

$$
A I=\frac{\Delta A_{\text {maj }}+\Delta A_{\text {min }}}{2 * A_{T}}
$$

Where differential $\Delta A$ is the smallest absolute area difference by overlapping about major and minor axis respectively and $A_{T}$ is the lesion area. If the Asymmetry is under 20\%, The lesion is symmetric. With a purpose to measure symmetry we rely on geometrical properties called second of inertia.

So far as asymmetry quantification is, beginning of the regional cartesian basis is the centre of mass $G$ of a present lesion $L$ described by a binary function $z(i, j), z(i, j)=1$ if $(i, j)$ $\in \mathrm{L}, 0$ otherwise. The centred moment $\mathrm{I}(\Phi)$,showing an angle $\Phi$ with the horizontal Cartesian, of the lesion $\mathrm{L}$ is given by eq. (2)

$$
I_{\phi}=\sum_{i, j \in L}(-i \sin \phi+j \cos \phi)^{2}=\sum_{i, j \in L} D_{\phi}(i, j)^{2}
$$

Where $D_{\Phi}(i, j)$ is the distance between the current pixel $I(i, j)$ and its projection on $\Delta$, along a direction normal to $\Delta$. The major axis is related to the smallest inertia moment of $\mathrm{L}$ and supplies the longitudinal path, $\varphi$ of L.

\subsubsection{Border Irregularity}

The Harris corner detection algorithm has been implemented for detect the notched and ragged edges. The border is divided into 8 regions and for every region the irregularities are marked and counted. The Harris corner detector isolates only small number of points which are most crucial for the diagnosis, is invariant to rotation, small changes of scale and image noise.

Let us assume a shift $(\Delta x, \Delta y)$ about a point $(x, y)$, then auto-correlation function could be expressed, $\mathrm{c}(\mathrm{x}, \mathrm{y})=\sum_{W}\left[\mathrm{I}\left(\mathrm{x}_{i}, \mathrm{y}_{i}\right)-\mathrm{I}\left(\mathrm{x}_{i}+\Delta \mathrm{x}, \mathrm{y}_{i}+\Delta \mathrm{y}\right)\right]^{2}$

Where $\mathrm{I}(\cdot, \cdot)$ denoted the image function and $\left(x_{i}, y_{i}\right)$ were the points in the Gaussian window $W$ centered on $(\mathrm{x}, \mathrm{y})$.

$$
c(x, y)=\left[\begin{array}{c}
\Delta x \\
\Delta y
\end{array}\right]^{T} S(x, y)\left[\begin{array}{c}
\Delta x \\
\Delta y
\end{array}\right]
$$

$S(x, y)$ represented the spatial intensity component in localized neighborhood of point $(x, y)$.

$S(x, y)$, for each point $I(x, y)$ in the input image was a matrix of size(2,2). Assume $\lambda_{1}$ and $\lambda_{2}$ be the eigen values associated to matrix $S(x, y)$. This selection and total dependency of Harris Detector on eigen values make it invariable to rotation as stated earlier.
The three important points resulted out by analyzing the eigen values of $S(x, y)$ are:-

a) Lesser values of both parameters resulted in flat points.

b) One smaller and one larger led to edge points.

c) High values of both of these eigen parameters defined as corner points in the image.

\subsubsection{Color Variagation}

In malignant melanoma there can be found up to six or even more different colors. Six colors are considered to be most significant: white, red, light-brown, dark-brown, blue gray, and black.

For showing the presence of 6 basic colors inside the lesion giving 1 score point to each and every for the calculation of the C score. Euclidian distance in "rgb" coordinates D is calculated between lesion color and the six reference colors mentioned above. For instance, for the primary colour, white $\mathrm{r} \lg 1 \mathrm{~b} 1=[1.0,1.0,1.0]$ and therefore:

$$
\begin{aligned}
& \mathrm{D}=\mathrm{SQRT}\left((\mathrm{r}-\mathrm{r} 1)^{2}+(\mathrm{g}-\mathrm{g} 1)^{2}+(\mathrm{b}-\mathrm{b} 1)^{2}\right) \\
& =\operatorname{SQRT}\left((\mathrm{r}-1.0)^{\wedge} 2+(\mathrm{g}-1.0)^{\wedge} 2+(\mathrm{b}-1.0)^{\wedge} 2\right)
\end{aligned}
$$

In our approach, we compare the distance $\mathrm{D}$ of the pixel under test, rgb, and the specified colour, to a somewhat arbitrarily chosen constant threshold value T. Exactness of colour matching depends upon the edge price $\mathrm{T}$. When the above approach is completed for all pixels within the lesion, percentages of color are calculated for each and every probably the most six colors. If the percent look of a specific colour is bigger than a targeted restrict $\mathrm{L}$ (e.G. $\mathrm{L}=0.45$ ), it's concluded that this colour is reward in the lesion and the $\mathrm{C}$ parameter is incremented by way of 1 , as much as a maximum value of 6 when all six colors are reward in the lesion.

\subsubsection{Equivalent Diameter}

In this we calculated the average of all possible diamegters in lesion passing through lesion's centre. Then the diameter value is converted into centimetre and then score points are assigned to the image, which is diameter $<=1.5 \mathrm{~cm}$ is given 1 point, diameter in the range of $1.5-2.5 \mathrm{cmis}$ given 2 point, diameter in the range $2.5-3.5 \mathrm{~cm}$ is given 3 point, $3.5-4.5 \mathrm{~cm}$ is given 4 points and more than 4.5 is given 5 points. Thus, Diameter score range lie between 1-5.

The processing techniques that were adopted for the feature extraction is then used to find Total Dermotoscopic score (TDS) with the equation below. If TDS is below 6.3 the lesion are interpreted as benign melanocytic lesion. If TDS is above 6.3 lesion is highly suggestive of melanoma.

TDS $=\left(\right.$ A_score*1.3) $+($ B_score* 0.1$)+($ C_score* 0.5$)+D \_s c o r e$ $* 0.5$ )

\section{EXPERIMENTAL SETUP AND RESULTS}

Tests of correct implementation of the dermoscopy ABCD rule method were carried out on the data sets of images, instances of which are depicted by Table4.1. The system has been tested on 184 lesions (144 malignant and 40 benign lesions). 
TABLE II. MELANOMA DATA SAMPLES

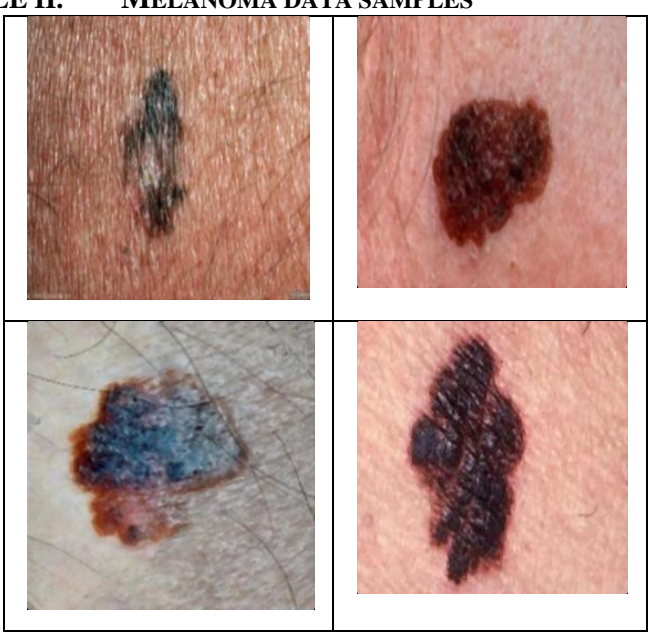

The result set for test image, retrieved from various operations of median filtering, segmentation and corners is depicted in Table.

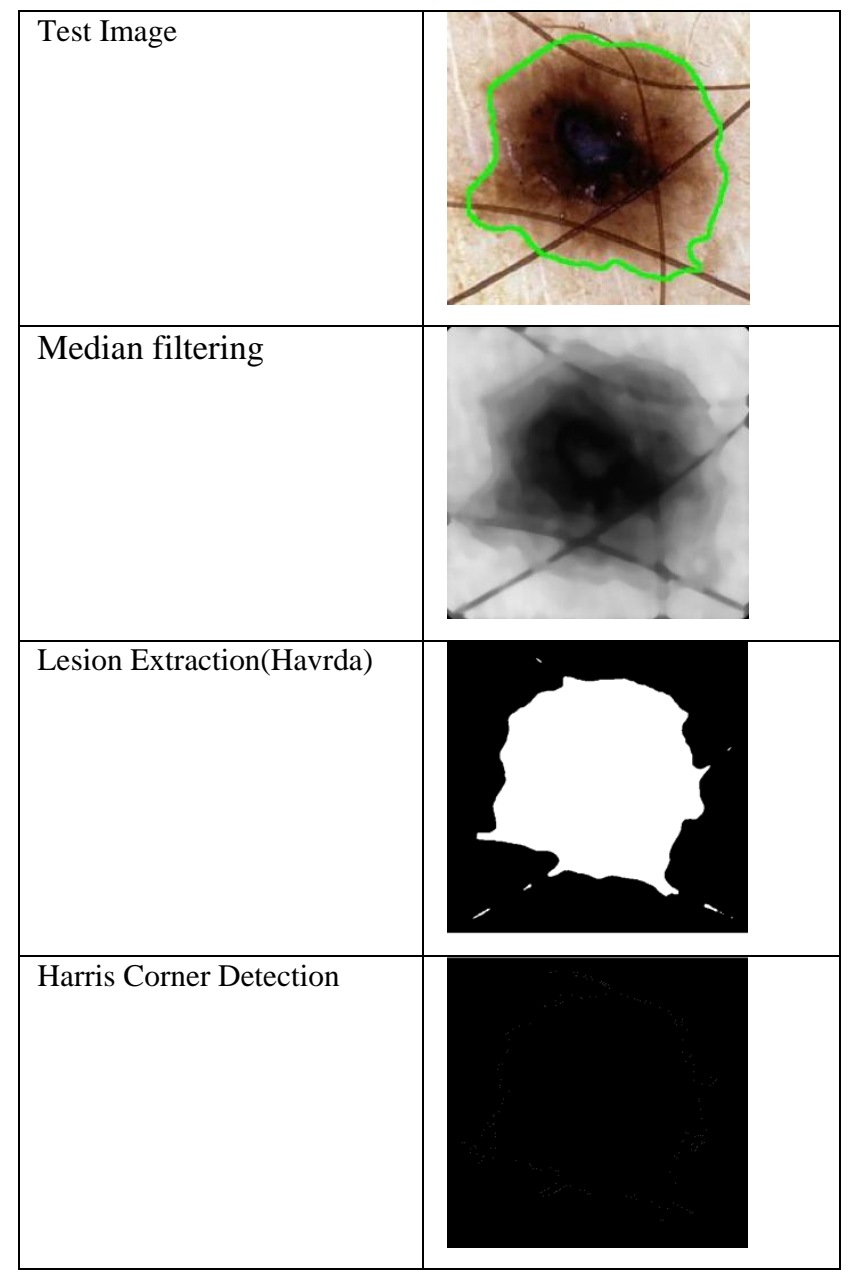

The resultant TDS score accounted using asymmetry, border irregularity, color variegation and diameter ranking is tabulated along with class labels in Table.

TABLE III: For Benign Image:

Entropy

$$
\text { Otsu }
$$

Segmentation:

Segmentation:

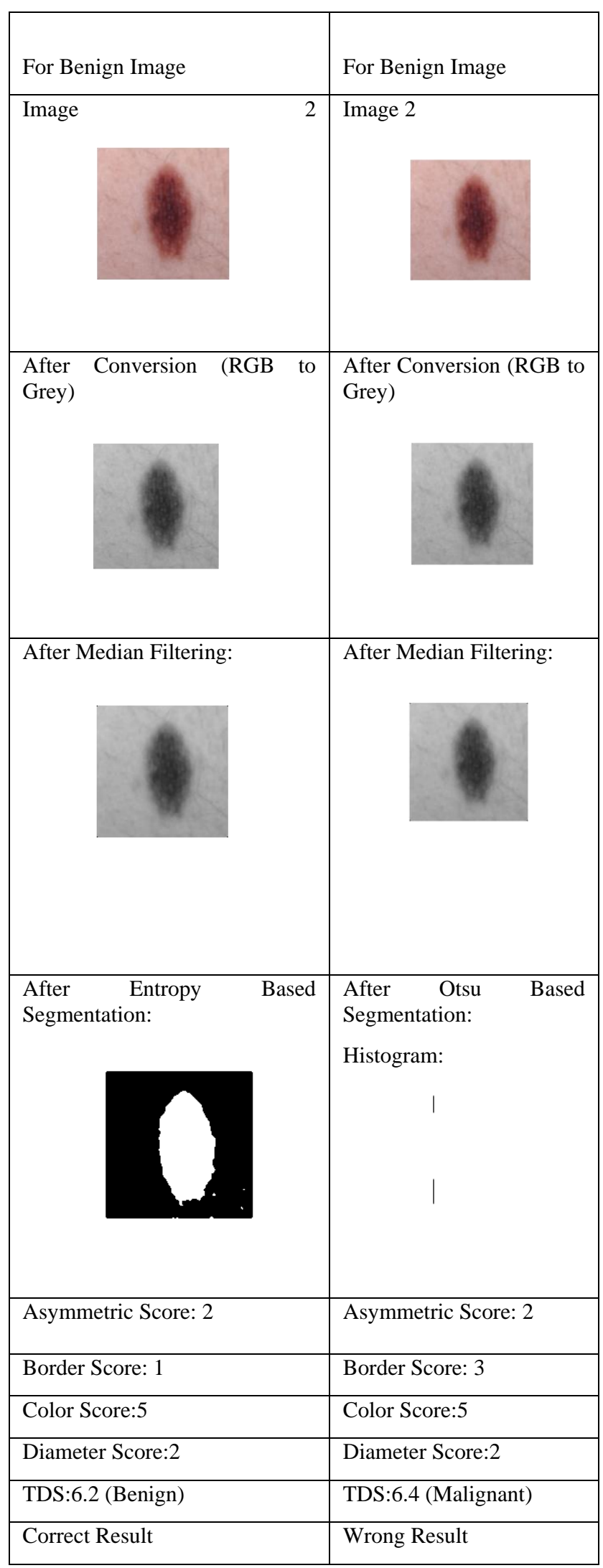


TABLE IV: For Malignant Image:

Entropy

Otsu

Segmentation:

Segmentation:

\begin{tabular}{|c|c|}
\hline For Malignant Image & For Malignant Image \\
\hline Image & Image 2 \\
\hline After Conversion (RGB to Grey) & $\begin{array}{l}\text { After Conversion (RGB } \\
\text { to Grey) }\end{array}$ \\
\hline After Median Filtering: & After Median Filtering: \\
\hline 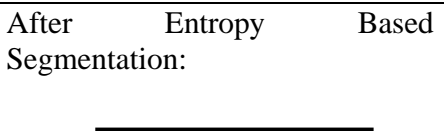 & $\begin{array}{l}\text { After Otsu Based } \\
\text { Segmentation: } \\
\text { Histogram: }\end{array}$ \\
\hline Asymmetric Score: 2 & Asymmetric Score: 2 \\
\hline Border Score: 6 & Border Score: 7 \\
\hline Color Score: 5 & Color Score: 3 \\
\hline Diameter Score: 2 & Diameter Score: 2 \\
\hline TDS:6.7 (Malignant) & TDS:5.8 (Benign) \\
\hline Correct Result & Wrong Result \\
\hline
\end{tabular}

The corresponding TDS plot for the whole image dataset considered against OTSU and Entropy based approach is graphically plotted in Figure 2 and 3 respectively.

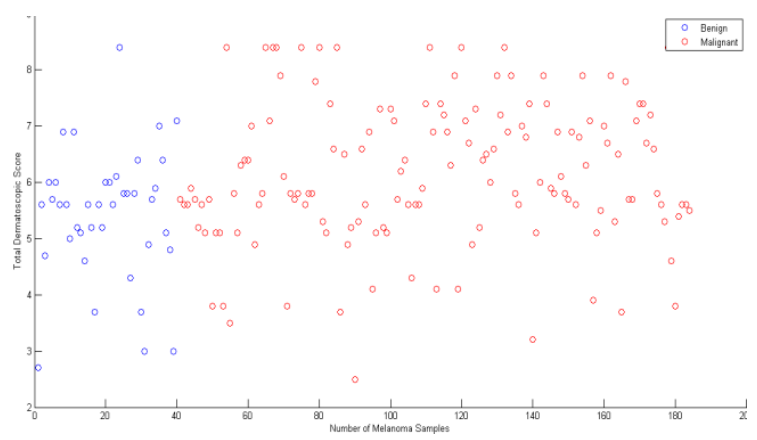

Fig 2. TDS Plot of proposed ABCD features using OTSU segmentation

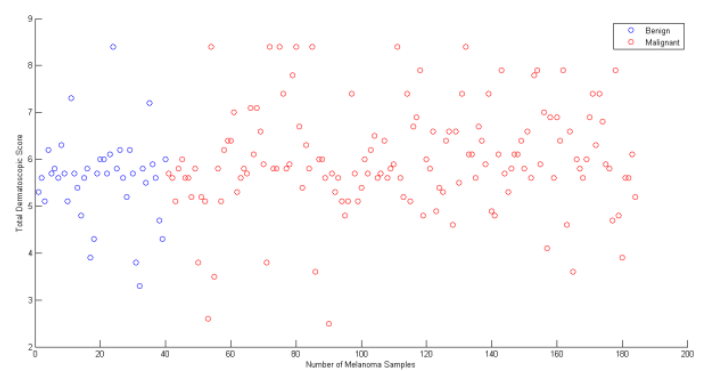

Fig3: TDS Plot of proposed ABCD features using HavrdaCharvat entropy

The specificity and sensitivity performance of both OTSU and entropy based melanoma diagnostic system is tabulated in Table 4, which proved that the Havrda based approach promisable results as compared to other investigated methods.

\section{TABLE III. SENSISTIVITY AND SPECIFICITY VALUES}

\begin{tabular}{|l|l|l|}
\hline \multicolumn{1}{|l|}{$\longrightarrow$} & $\begin{array}{l}\text { Otsu with Harris } \\
\text { Corner (ABCD } \\
\text { features) }\end{array}$ & $\begin{array}{l}\text { Havrdarith } \\
\text { Harris } \begin{array}{r}\text { Corner } \\
\text { (ABCD features) }\end{array} \\
\text { Performance }\end{array}$ \\
\hline Sensitivity & $90.41 \%$ & $92.45 \%$ \\
\hline Specificity & $29.72 \%$ & $27.48 \%$ \\
\hline
\end{tabular}

The Extracted features at a trained using the Neural Networks $(\mathrm{N}-\mathrm{N}) .70 \%$ of input data is considered for training the classifier. Rest $30 \%$ is categorized for validation and testing set of $15 \%$ each. The experimental outcome for $\mathrm{N}-\mathrm{N}$ classifier is shown as below: 

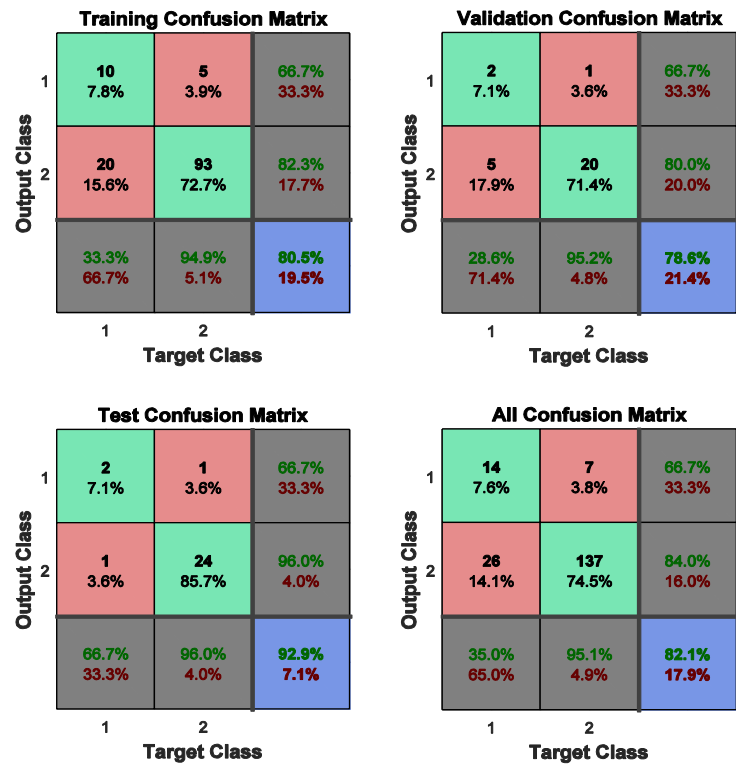

Figure no.4 Simulation outcomes of Neural Network Classifier

TABle IV. Sensistivity AND Specificity Values USING NEURAL NETWORK

\begin{tabular}{|l|l|l|}
\hline \multicolumn{1}{|l|}{$\longrightarrow$} & $\begin{array}{l}\text { Otsu with Harris } \\
\text { Corner (ABCD } \\
\text { features) }\end{array}$ & $\begin{array}{l}\text { Havrda with } \\
\text { Harris Corner } \\
\text { (ABCD features) } \\
\text { Approaches } \\
\text { Performance }\end{array}$ \\
\hline Sensitivity & $95.13 \%$ & $99.30 \%$ \\
\hline Specificity & $7.5 \%$ & $0 \%$ \\
\hline
\end{tabular}

The Neural network classifier proves the significance of image segmentation approach deployed for melanoma dyganosis. OTSU Method shows the sensitivity of $95.13 \%$ and specificity of $7.5 \%$. while Entropy base method shows the sensitivity of $99.30 \%$ and specificity of $0 \%$. Thus in the case with $100 \%$ confident for true positiveness (Malignancy) the entropy base approach is adventurous while in the case of general diagnostic system with the lower false negative ratio the otsu entropy base diagnosis performs well.

\section{CONCLUSION}

In the thesis, we attempted to diagnose melanoma from color skin images using image processing techniques. Achieved results indicate that the application can be used for the diagnosis of malignant melanomas. The features Asymmetry, Border, Color variation and Diameter are used for the calculation of Total Dermatoscopic Score (TDS). A high TDS score greater than 5.9 means lesion is more likely to malignant melanoma. In future this work can be extended to investigate further on enlarged database so as to hypothesize diagnostic model on the melanoma population.

\section{REFERENCES}

[1] W. Barhoumi, E. Zagrouba, "A Prelimary Approach for The Automated Recognition of malignant Melanoma",Image Anal Stereol, pp.121-135, 2004.
[2] EzzeddineZagrouba and WalidBarhoumi, "A prelimary approach for the automated reconition of malignant melanoma", Image Anal Stereol, 23:121-13, 2004

[3] David Houcque, "Introduction to MATLAB for Engineering Students", Northwestern University, version 1.2 , August 2005

[4] G. grammatikopoulos, A. Hatzigaidas, A. Papastergiou, P. Lazaridis, Z. Zaharis, D. Kamptaki, G. Tryfon, "Automated Malignant Melanoma Detection Using MATLAB", International Conference on Data Networks, Communications \& Computer Proceedings of the 5th WSEAS, Bucharest, Romania, October 16-17, 2006

[5] Dave Tahmoush and HananSamet, "Using Image Similarity and Asymmetry to Detect Breast Cancer", Medical Imaging, Proc. of SPIE Vol. 6144, 61441S, (2006)

[6] F. Mai, Y. Hung, H. Zhong, and W. Sze, “A hierarchical approach for fast and robust ellipse extraction",Pattern Recognition, 41(8):2512-2524, August 2008

[7] Andreas Blum, Iris Zalaudek, "Digital Image Analysis for Diagnosis of Skin Tumors", Seminar in Cutaneous Medicine and Surgery, Elsevier Inc,27:11-15 () 2008

[8] Shekhar Singh, Dr P. R. Gupta, "Breast Cancer detection and classification using Neural Network", International Journal of Advanced Engineering Sciences and Technologies, vol no. 6, issue no. 1, 004, 2009

[9] Michael D. Stubblefield, Michael, "Cancer rehabilitation principles and practice", Demos ", International Journal of Computer Theory and Engineering, Vol. 1, No. 5, December, 2009, 1793-8201.

[10] Abbas, Q.; Celebi, M.; García, I. F. Skin tumor area extraction using an improved dynamic programming approach. Skin Research and Technology, 2012, v. 18(2): p. 133-142.

[11] Alcón, J. F.; Ciuhu, C.; Kate, W. T.; Heinrich, A.; Uzunbajakava, N.; Krekels, G.; Siem, D.; Haan, G. d. Automatic imaging system with decision support for inspection of pigmented skin lesions and melanoma diagnosis. IEEE Journal of Selected Topics in Signal Processing, 2009, v. 3(1): p. 14-25.

[12] Argenziano, G.; Fabbrocini, G.; Carli, P.; Giorgi, V. D.; Sammarco, E.; Delfino, M. Epiluminescence microscopy for the diagnosis of doubtful melanocytic skin lesions: Comparison of the abcd rule of dermatoscopy and a new 7-point checklist based on pattern analysis. Archives of Dermatology, 1998, v. 134(12): p. 1563- 1570.

[13] Castiello, C.; Castellano, G.; Fanelli, A. M. Neuro-fuzzy analysis of dermatological images, in IEEE International Joint Conference on Neural Networks. 2004.

[14] Skin Cancer Facts With Statistics", National council on skin cancer prevention ,Friday May 24, 2013.

[15] Barhoumi \& E. Zagrouba, "A Prelimary Approach For The Automated Recognition Of Malignant Melanoma," Image AnalStereol, pp. 121-135, 2004.

[16] W. Barhoumi \& E. Zagrouba in: E.Damiani, R.J. Howlett, L.C. Jain, N.Ichalkaranje (Eds.), Boundaries Detection Based on Polygonal Approximation by Genetic Algorithms, Knowledge-Based Intelligent 
Information Engineering Systems and Allied Technologies (KES 2002), Frontiers in artificial intelligence and applications, IOS Press 82(2), Amsterdam, pp. 621-7, 2002.

[17] Maciej Ogorzalek, Leszek Nowak, Grzegorz Surówka and Ana Alekseenko,"Modern Techniques for computeraided melanoma diagnosis",ISBN:978-953-307-571-6, Intech 2011

[18] G. Grammatikopoulos, A. Hatzigaidas, Papastergiou, P. Lazaridis, Z. Zaharis, D. Kampitaki, G. Tryfon, "Automated Malignant Melanoma Detection Using MATLAB", Proceedings of the 5th WSEAS Int. Conf. on DATA NETWORKS, COMMUNICATIONS \& COMPUTERS, Bucharest, Romania, October 16-17, 2006.

[19] Gautam,D.; Ahmed, M., "Melanoma Detection and Classification Using SVM Based Decision Support System “, in IEEE INDICON 2015, 17-20 December 2015.

[20] Singh, D.; Gautam, D.; Ahmed, M., "Detection techniques for melanoma diagnosis: A performance evaluation," in Signal Propagation and Computer Technology (ICSPCT), 2014 International Conference on , vol., no., pp.567-572, 12-13 July 2014. 